\title{
Analysis of 3-Level Bandpass Sigma-Delta Modulators With 2-Level Output
}

\author{
Tomasz Podsiadlik, John Dooley, and Ronan Farrell
}

\begin{abstract}
This brief analyzes a recently introduced, 2-level bandpass modulator incorporating 3-level lowpass $\Sigma \Delta$ modulators. While the combination of 3-level $\Sigma \Delta$ with 2-level output improves output signal-to-noise ratio (SNR) in a wideband sense, it also introduces nonlinear distortion in the 2-level output. In this brief, the nonlinear effects are modeled and a linearization technique offering up to $22 \mathrm{~dB}$ of error cancellation is derived. In result, the modified 2-level modulator can show up to $6 \mathrm{~dB}$ of an SNR improvement versus equivalent for a 2 -level $\Sigma \Delta$ modulator. The new technique is demonstrated through analysis and experimental results.
\end{abstract}

Index Terms-Field-programmable gate array (FPGA), linear model, predistortion, sigma delta.

\section{INTRODUCTION}

A BANDPASS delta-sigma modulator $(\mathrm{BP} \Sigma \Delta \mathrm{M})$ utilizing the error-pulse shaping has been introduced in [1]. This new modulation technique uses a pair of 3-level lowpass $\Sigma \Delta$ modulators ( $\mathrm{LP} \Sigma \Delta \mathrm{M}$ ) followed by discrete time mixer and a 2-level quantizer. The new 2-level modulator offers up to $6 \mathrm{~dB}$ of signal-to-noise ratio (SNR) improvement in a wideband sense when compared with a conventional 2-level modulator. This is achieved by employing 3-level $\operatorname{LP} \Sigma \Delta \mathrm{M}$. Unlike the known approach, employing pulse width modulation [2] for representing a multilevel signal by a 2-level signal, the new technique is based on suppression of the nonlinear effects at the frequency of interest. Namely, the modulator output is considered as a three level signal, whose "zero" level is offset such that it has the same value as the upper or lower quantizer level. While the offsetting action creates distortion in the output of the modulator, a discrete time Fourier transform (DTFT) calculated for the error pulse has a single zero which reduces the distortion effect at the frequency of interest. Although the single zero is sufficient for disguising the impact of the distortion in a first order $\Sigma \Delta \mathrm{M}$, the degradation in SNR becomes more apparent in outputs of second, third, or higher order modulators. This brief analyzes the mechanism of the distortion in the modulator introduced in [1], and demonstrates that an inverse

Manuscript received July 6, 2016; revised November 10, 2016; accepted November 29, 2016. Date of publication December 13, 2016; date of current version March 26, 2018. This work was supported by the Science Foundation Ireland under Grant 03/CE3/I405. This brief was recommended by Associate Editor T. B. Tarim.

T. Podsiadlik is with Benetel Ltd., D08 AT8K Dublin, Ireland (e-mail: tomaszpodsiadlik@benetel.com).

J. Dooley and R. Farrell are with the National University of Ireland Maynooth, W23 F2H6 Maynooth, Ireland (e-mail: john.dooley@nuim.ie; ronan.farrell@nuim.ie).

Color versions of one or more of the figures in this paper are available online at http://ieeexplore.ieee.org.

Digital Object Identifier 10.1109/TCSII.2016.2639282

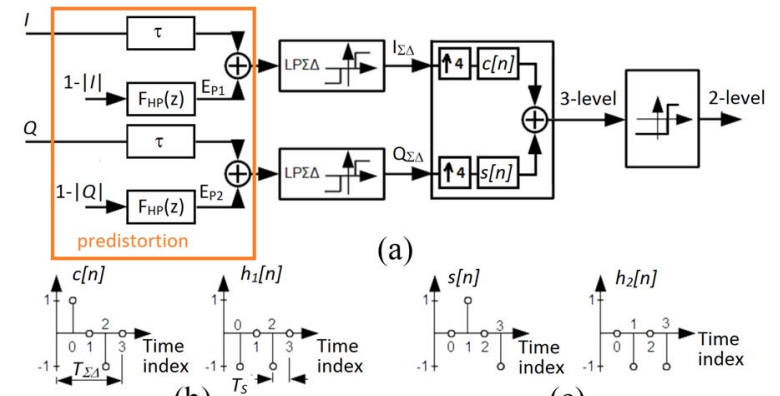

(b)

(c)

Fig. 1. (a) Two-level modulator employing 3-level LP $\Sigma \Delta$ M. (b) Carrier $c[n]$ and corresponding error pulse $h_{1}[n]$. (c) Carrier $s[n]$ and corresponding error pulse $h_{2}[n]$.

function can be employed for further reduction of the error magnitude.

\section{LinEAR MODEL OF THE MODUlatoR}

The modulator described in the continuous (CT) time domain in [1] is presented in the discrete time (DT) domain in Fig. 1(a). It consists of two DT inputs denoted in a similar fashion as in wireless transceivers, $I$ and $Q$. It should be noted that the application of the modulator is not limited to wireless communication signals only, and it can be easily used as a BP $\Sigma \Delta \mathrm{M}$ having the signal band at one fourth of the output sampling frequency [3]. The blocks $F_{\mathrm{HP}}(z)$ and $\tau$ are used for error cancellation which will be described later in Section III of this brief. All analysis made in Section II assumes that the predistortion block is omitted, and both, $I$ and $Q$ signals are fed directly to LP $\Sigma \triangle \mathrm{M}$ inputs. The first part of the modulation occurs in the 3-level LP $\Sigma \Delta \mathrm{M}$, whose quantizers have levels " $+A$," "0," and " $-A$." Subsequently, both 3-level outputs are upsampled by factor of four and mixed with $90^{\circ}$ phase shifted carriers, $c[n]$ and $s[n]$ shown in Fig. 1(b) and (c). This creates a bandpass signal at the sampling frequency of the LP $\Sigma \Delta \mathrm{M}$ denoted as $f_{\Sigma \Delta}$. An observation has been made in [1] that when $I_{\Sigma \Delta}=0$, both zero-valued samples at indexes 0,2 in $c[n]$ can be offset by the same positive or negative amplitude, which is equivalent to the addition of error pulse $h_{1}[n]$ as shown Fig. 1(b). Similarly when $Q_{\Sigma \Delta}=0$, zero-valued samples at indexes 1,3 in $s[n]$ can be offset creating the error pulse $h_{2}[n]$, as shown Fig. 1(c). Furthermore, the DTFT of any of these two error pulses has a single zero at the frequency $f_{\Sigma \Delta}$, which guarantees suppression of the error induced by $h_{1}[n]$ and $h_{2}[n]$ at the frequency of interest. Consequently, all zero 


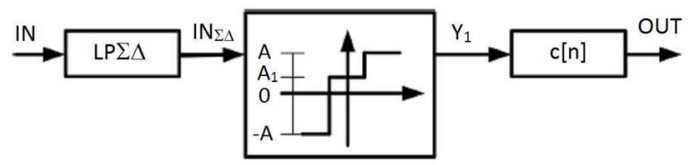

Fig. 2. Rearranged modulator used for calculating the input-output transfer characteristic.

valued outputs can be offset to match with the $+A$ or $-A$ levels at the cost of small degradation in the bandpass signal quality as described in [1]. This property is utilized to transform the 3-level $\operatorname{LP} \Sigma \Delta$ outputs to 2-level waveform in the modulator of Fig. 1(a). The resulting output has the property of a 3-level modulated signal, i.e., up to $6 \mathrm{~dB}$ less of the quantization noise than a 2-level $\Sigma \Delta \mathrm{M}$ output, while the 2-level output does not suffer the linearity problem typical to a multilevel $\Sigma \Delta$ digital to analog converter (DAC). The drawback of the new modulator is in the distortion introduced in the course of the 2-level quantization, whose effects are not entirely removed by the single zero in the DTFT of the error pulses. This problem is described in detail in the following sections.

\section{A. Transfer Characteristic of the Modulator}

For the purpose of analysis it is convenient to consider the 2-level modulator independently for $I$ and $Q$ signals. Such division into two subsystems allows for calculating the transfer-characteristics of the modulator and estimating the magnitude of the error added in its output. Accordingly, let us assume that only one of the two input signals is supplied to the modulator of Fig. 1(a), while the other one is zero. Also, without affecting the output of the modulator, the quantizer with zero-level offset can be placed ahead of the mixer as is depicted in Fig. 2. The quantizer in the rearranged modulator scheme adds the offset $A_{1}$ to the output $Y_{1}$ every time the 3-level signal $\mathrm{IN}_{\Sigma \Delta}$ is zero. For slowly changing input IN the average quantizer output $Y_{1}$ becomes

$$
Y_{1}=\mathrm{IN}+E_{\mathrm{IN}}
$$

The average value of the error added to IN depends on the probability of quantizer output being zero, $\operatorname{Pr}(0)$

$$
E_{\mathrm{IN}}=\operatorname{Pr}(0) A_{1} .
$$

Let us assume that for every positive input, a 3-level output of a first order $\operatorname{LP} \Sigma \Delta \mathrm{M}$ switches between two levels, 0 and $+A$ and the average output equals to slowly changing input IN. Similar, for a negative input, the 3-level output of the same modulator switches between 0 and $-A$, while maintaining the average output same as the input IN. Applying these properties and assuming that $-A<\mathrm{IN}<+A$, the zero output probability becomes

$$
\operatorname{Pr}(0)=1-|\mathrm{IN}| / A
$$

substituting (3) in (2) and in (1) yields

$$
\begin{aligned}
E_{\mathrm{IN}} & =A_{1}-|\mathrm{IN}| A_{1} / A \\
Y_{1} & =\mathrm{IN}+A_{1}-|\mathrm{IN}| A_{1} / A .
\end{aligned}
$$

Since $E_{\mathrm{IN}}$ is the average value of the 2-level waveform of amplitude $A_{1}$, it is also associated with a quantization

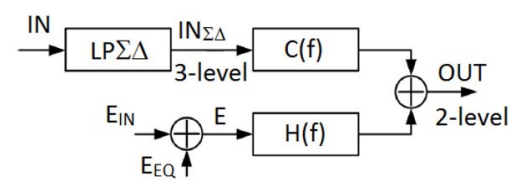

Fig. 3. Linear model of the modulator.

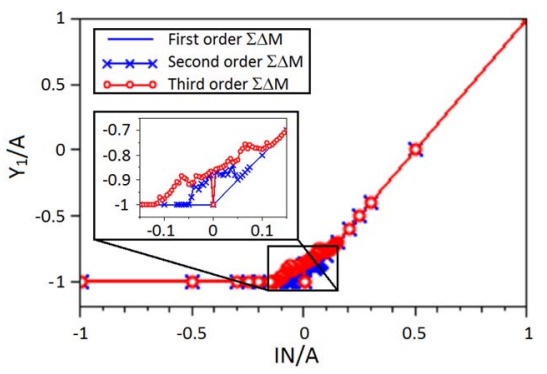

Fig. 4. Transfer characteristic of the modulator based on simulation of $\operatorname{Pr}(0)$.

error $E_{\mathrm{EQ}}$. Using similar reasoning as for $E_{\mathrm{IN}}$, the power of error $E_{\mathrm{EQ}}$ can be expressed as a function of the input IN

$$
E_{\mathrm{EQ}}^{2}=\left(A_{1}^{2} / A\right)\left(|\mathrm{IN}|-|\mathrm{IN}|^{2} / A\right) .
$$

As a result of mixing with the carrier, the error signals $E_{\mathrm{IN}}$ and $E_{\mathrm{EQ}}$ are suppressed by zeros in the functions $H_{1}\left(e^{j \omega}\right)$ and $\left.H_{2}{ }^{j \omega}\right)$, being DTFTs from $h_{1}[n]$ and $h_{2}[n]$, which reduces the nonlinear effect added in the 2-level quantizer. The linear model of the modulator of Fig. 1(a) based on the above analysis is shown in Fig. 3.

\section{B. Simulated Transfer Characteristic of the Modulator}

The analysis performed in Section II-A assumes that a first order, 3-level LP $\Sigma \Delta \mathrm{M}$ supplied with a slowly changing positive input, yields two levels in the quantizer output, i.e., $+A$ or 0. Similar, when the modulator is supplied with negative input, it can respond with output having one of two levels, $-A$ or 0 . This assumption is not true over the entire range of the input IN in higher order modulators. It is observed that when a slowly changing input approaches zero, the output of a 3-level LP $\Sigma \Delta \mathrm{M}$ can take any of the three values, $-A, 0$ or $+A$. The range of IN for which this effect occurs depends on the noise transfer function (NTF) of a LP $\Sigma \Delta$ M. In general, the higher order $\operatorname{LP} \Sigma \Delta \mathrm{M}$ used, the wider is the range which is not in agreement with $Y_{1}$ described by (5). Transfer input-output relationships based on simulation of $\operatorname{Pr}(0)$ of first to third order cascaded integrators with distributed feedback (CIFB) [2] modulators are shown in Fig. 4. The zeros and poles of NTFs used to simulate $\operatorname{Pr}(0)$ are listed in Table I. It is observed that only $Y_{1}$ of the first order modulator is in a agreement with (5). On the other hand, modulators of second and third order do not follow the zero output probability (3) at low amplitudes of IN. The correction of characteristic of second and third order modulators will be described in Section II-C.

\section{Nonlinear Distortion}

The modulator model of Fig. 3 can be used for predicting of distortion in the output of the 2-level modulator. A sine waveform is a convenient signal for investigating effects of 
TABLE I

NTF Zeros and Poles. Both Modulators Used $\left\|\mathrm{NTF}_{\infty}\right\|=1.5$

\begin{tabular}{|c|c|c|}
\hline Ord & Zeros & Poles \\
\hline 2 & $z_{1,2}=1$ & $p_{1,2}=0.61 \pm j 0.26$ \\
\hline 3 & $z_{1,2,3}=1$ & $\begin{array}{c}p_{1,2}=0.77 \pm j 0.28 \\
p_{3}=0.67 ;\end{array}$ \\
\hline
\end{tabular}

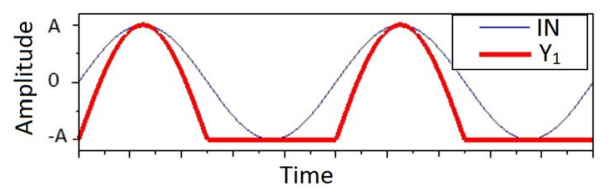

Fig. 5. Input-output waveforms.

the distortion. If a zero-level offset in modulator of Fig. 2 is $A_{1}=-A$ then, according to the input-output relationship of Fig. 4, the output of the modulator will be closely approximated by the half-wave rectified sine wave depicted in Fig. 5. Apart from the harmonics resulting from addition of the error $E_{\mathrm{IN}}$, the output will carry quantization error $E_{\mathrm{EQ}}$ as explained in Section II-A. The behavior of $Y_{1}$ at low input values seen in Fig. 4 may also be included in the model of the modulator for better accuracy. The value of the $\operatorname{Pr}(0)$ at low IN and the range of the input has been obtained by simulating a 3-level LP $\Sigma \Delta \mathrm{M}$ supplied with a set of constant inputs. Modulators in this brief use $\operatorname{Pr}_{2}(0)=0.9$ when $-0.045<$ IN $<0.045$ and $\operatorname{Pr}_{3}(0)=0.86$ when $-0.125<$ IN $<0.125$ in the case of second and third order, respectively. When outside the low amplitude range, probability is described by (3). A simple approximation of the transfer function by (2) yielded approximately $4 \mathrm{~dB}$ discrepancy between the modeled and actual modulator output at higher harmonics when using second order LP $\Sigma \Delta \mathrm{M}$. This difference was largely removed after setting a fixed value of $\operatorname{Pr}(0)$ as has been described above. After upsampling and mixing, both $E_{\mathrm{IN}}$ and $E_{\mathrm{EQ}}$ are suppressed at frequency $f_{\Sigma \Delta}$ by $H_{1}\left(e^{j \omega}\right)$ as it is seen in Fig. 6. Since $E_{\mathrm{IN}}$ error is deterministic, it becomes a subject of linearization in the following sections of this brief.

\section{LINEARIZATION}

The errors $E_{\mathrm{IN}}$ and $E_{\mathrm{EQ}}$ are attenuated in the output of the modified 2-level modulator by function $H_{1}\left(e^{j \omega}\right)$ or $H_{2}\left(e^{j \omega}\right)$. A fraction of these errors, however, fall into the signal band. This section describes a linearization function derived for the 2-level modulator which is used to reduce the deterministic error $E_{\mathrm{IN}}$. The linearization, however, does not reduce the effect of quantization error $E_{\mathrm{EQ}}$.

\section{A. Predistortion Function}

Let us assume that the error cancellation signal denoted by $E_{P}$ is added directly to the modulator's input $I$ as it is shown in Fig. 1(a), while the input $Q$ remains zero. Consequently, the input to the upper LP $\Sigma \Delta \mathrm{M}$ becomes $I+E_{P 1}$. This input signal is next transferred to the frequency $f_{\Sigma \Delta}$ as a result of mixing with the pulse $c[n]$. The error $E_{\mathrm{IN}}$ appears in the output of the modulator suppressed by $H_{1}(z)$, being $Z$-transform of $h_{1}[n]$. Using the linear model of the modulator of Fig. 3, modulator

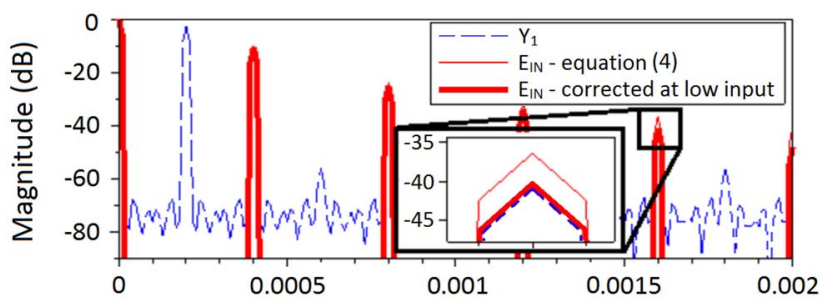

(a)

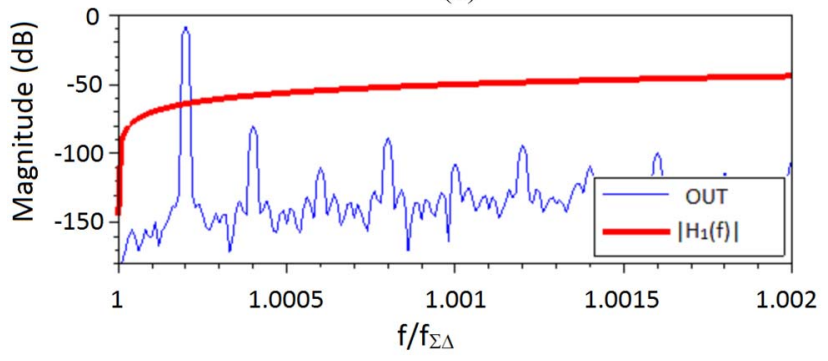

(b)

Fig. 6. Simulation results for second order $\Sigma \Delta \mathrm{M}$. (a) Output $Y_{1}$ and modeled error $E_{\mathrm{IN}}$. (b) Modulator output at frequency $f_{\Sigma \Delta}$.

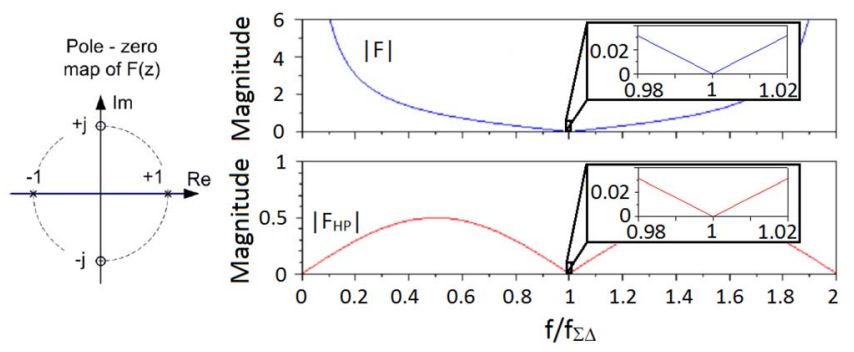

Fig. 7. Magnitude responses $|F(f)|$ and $\left|F_{\mathrm{HP}}(f)\right|$ for $g=0.25$.

output can be expressed as

$$
\operatorname{OUT}(z)=\left[I(z)+E_{P 1}(z)\right] C(z)+\left[E_{\mathrm{IN}}(z)+E_{\mathrm{EQ}}(z)\right] H_{1}(z) .
$$

The role of $E_{P 1}$ is to cancel the error $E_{\mathrm{IN}}$ thus

$$
\begin{aligned}
& E_{P 1}(z) C(z)+E_{\mathrm{IN}}(z) H_{1}(z)=0 \\
& E_{P 1}(z)=-\frac{E_{\mathrm{IN}}(z) H_{1}(z)}{C(z)}=E_{\mathrm{IN}}(z) F(z) .
\end{aligned}
$$

The signal $E_{P 1}$ is obtained by using filter function $F(z)$ and error signal $E_{\mathrm{IN}}(z)$. Using $Z$-transforms to express $H_{1}(z)$ and $C(z)$ in (9) yields

$$
F(z)=\frac{1+z^{-2}}{1-z^{-2}} .
$$

The transfer function $F(z)$ has zeros at $z= \pm j$, and poles on a unit circle at $z= \pm 1$, which results in the magnitude of $F(z)$ tending to infinity when the frequency approaches $f=0$ and $f=2 f \Sigma \Delta$, as shown in Fig. 7. The phase response is constant, $+\pi$ or $-\pi$, implying zero group delay. The transfer function $F(z)$ cannot be used to directly implement the desired filter at the inputs of the modulator of Fig. 1. It is because, as shown in Fig. 7 the period of the frequency response of $F(z)$ is $2 f_{\Sigma \Delta}$, while sampling frequency of both $\Sigma \Delta \mathrm{M}$ is only $f_{\Sigma \Delta}$. The transfer function $F(z)$ can be, however, approximated within the narrow band of interest centered at the carrier frequency 

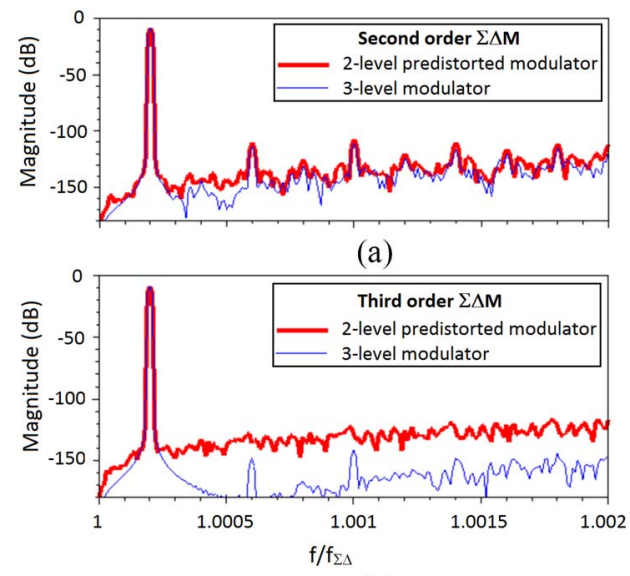

(b)

Fig. 8. Simulated spectra of predistorted 2-level and 3-level modulators outputs. (a) Second order $\Sigma \Delta \mathrm{M}$. (b) Third order $\Sigma \Delta \mathrm{M}$.

$f_{C}=f_{\Sigma \Delta}$ by another transfer function, whose period of repetition is $f_{\Sigma \Delta}$. Let us denote this approximating transfer function by $F_{\mathrm{HP}}(z)$. The magnitude response of $F_{\mathrm{HP}}(z)$ must be very similar to that of $F(z)$ in the proximity of $f_{C}=f_{\Sigma \Delta}$. Using a first order high-pass filter we can obtain a single zero at frequency $f_{\Sigma \Delta}$ similar as $F(z)$. In addition, both functions $F(z)$ and $F_{\mathrm{HP}}(z)$ have magnitude responses which can be approximated by linear function over narrow frequency range in the proximity of frequency $f_{\Sigma \Delta}$. Let us write that

$$
F_{\mathrm{HP}}(z)=g\left(1-z^{-1}\right)
$$

where $g=0.25$ scales the magnitude response of the filter. For the purpose of simulation an approximation bandwidth (BW) of $f_{\Sigma \Delta}(1 \pm 0.02)$ corresponding to oversampling ratio $\mathrm{OSR}=25$ of $\mathrm{LP} \Sigma \Delta \mathrm{M}$ was chosen. Magnitude responses of both functions are shown for comparison in Fig. 7, having nearly the same values in proximity of frequency $f_{\Sigma \Delta}$. Since the function $F_{\mathrm{HP}}(z)$ has group delay of $\tau=\pi / 4$, a delaying filter denoted by $\tau$ is also added in the input branch in Fig. 1(a). In a practical implementation, a simple first-order filter can be used in this purpose, e.g., $F_{\mathrm{LP}}(z)=\left[\left(1+z^{-1}\right) / 2\right]$, as it is done in this brief.

\section{B. Predistortion in First, Second, and Third Order $\Sigma \Delta M$}

The effects of linearization are simulated using first, second and third order modulators, supplied with a single tone signal of frequency $f_{0}=2 \cdot 10^{-4} f_{\Sigma \Delta}$. All simulated modulators used in this brief are CIFB structures [2], characterized by quantizer full scale (FS) range $\Delta=2 A$. The input tone is scaled to occupy 0.75 of FS, which ensures that none of the modulators is overloaded. The second and third order $\Sigma \Delta \mathrm{M}$ have zeros and poles listed in Table I. In order to obtain best cancellation of $E_{\mathrm{IN}}$, the $\operatorname{Pr}(0)$ is modeled at higher input amplitudes by (3), and at low input amplitude values by constants as described in Section II-C. The simulated output spectrum of second order $\Sigma \Delta \mathrm{M}$ is shown in Fig. 8(a). An observation is made that the output of the linearized modulator is free of the artifacts at frequencies $f_{\Sigma \Delta}+2 f_{0}, f_{\Sigma \Delta}+4 f_{0}, \ldots, f_{\Sigma \Delta}+10 f_{0}$ previously observed in Fig. 6 in the output of the modulator without

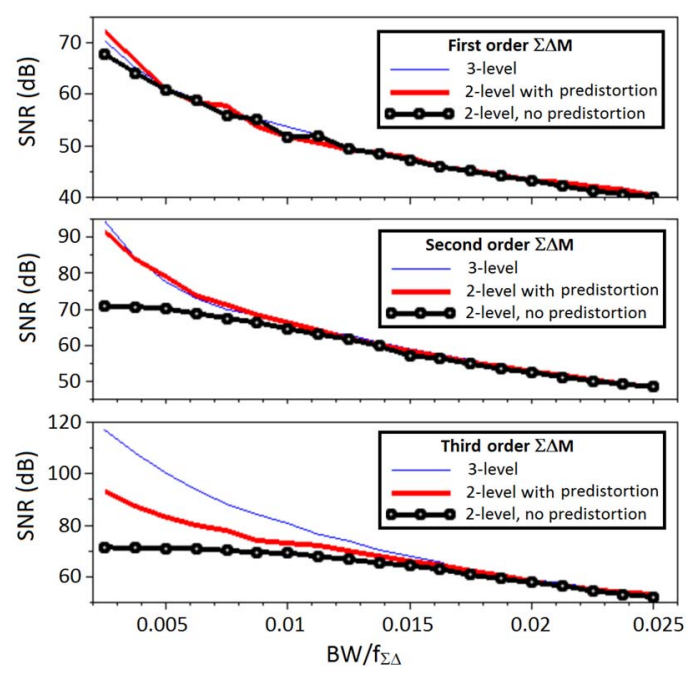

Fig. 9. Simulated SNR of first, second and third order modulators.

linearization. In addition, the 2-level output shows nearly as good SNR as the 3-level modulator also shown in Fig. 8(a).

Third order, 2-level modulator whose output spectrum is shown in Fig. 8(b) also shows no evidence of nonlinear distortion. However, when compared against the 3-level modulator, the latter shows better SNR. While the quantization error of the 3-level $\Sigma \Delta \mathrm{M}$ is shaped by the third order NTF, $E_{\mathrm{EQ}}$ is shaped by first order function $H(z)$. This weaker suppression of $H(z)$ manifests itself in the increased noise level in comparison to 3-level modulator as seen in Fig. 8(b).

The simulated SNR as function of BW are shown in Fig. 9. The SNR was calculated by supplying the modulators with the same single tone input, while changing the BW. As could be predicted, the 2-level and 3-level modulators show similar SNR performance at higher BW, where the dominant is the quantization noise of the 3-level $\Sigma \Delta \mathrm{M}$. The difference between 2-level and 3-level modulators becomes observable when dominant are errors $E_{\mathrm{IN}}$ and $E_{\mathrm{EQ}}$. This occurs when BW is less than $5 \cdot 10^{-3} f_{\Sigma \Delta}$, less than $11 \cdot 10^{-3} f_{\Sigma \Delta}$ and less than $16 \cdot 10^{-3} f_{\Sigma \Delta}$ for first, second and third order $\Sigma \Delta \mathrm{M}$, respectively. While the use of linearization shows benefit of $6 \mathrm{~dB}$ of SNR in the first order, 2-level modulator, it is observed that the linearization in second and third order modulators improves the SNR by $22 \mathrm{~dB}$ when $B W \leqslant 2.5 \cdot 10^{-3} f_{\Sigma \Delta}$. In all three cases the SNR in the modulator with no predistortion achieves maximum value of approximately $71 \mathrm{~dB}$. This SNR limitation is caused by the harmonics at frequencies $f_{\Sigma \Delta}+2 f_{0}$, $f_{\Sigma \Delta}+4 f_{0}, \ldots$, seen in Fig. 6, which become dominating distortion components for low BW.

In addition to 1-tone, the predistortion was simulated with a 9-tone input. After adding the predistortion, the SNR calculated over signal and adjacent channel BW showed $8.5 \mathrm{~dB}$ of SNR improvement for second and third order modulators at $\mathrm{OSR}=260$. The predistorted modulators of second and third order had SNR lower than their 3-level counterparts by $7.5 \mathrm{~dB}$ and $35 \mathrm{~dB}$, respectively. The large SNR degradation in the third order 2-level modulator output was caused by presence $E_{\mathrm{EQ}}$, which was also observed earlier for 1-tone input. In the two simulated cases, SNR differences between 


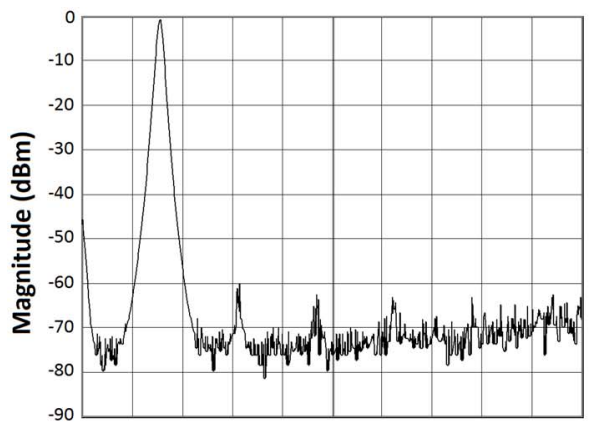

(a)

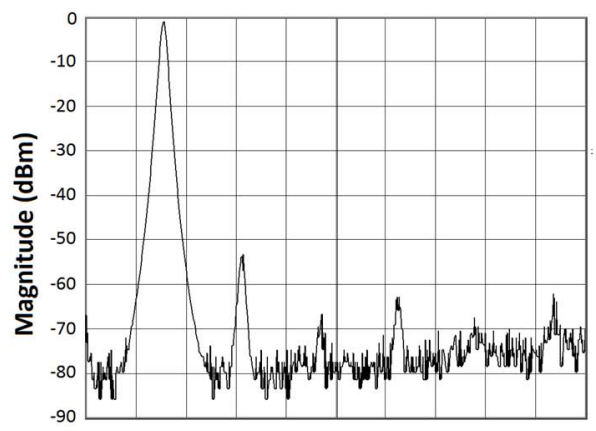

(b)

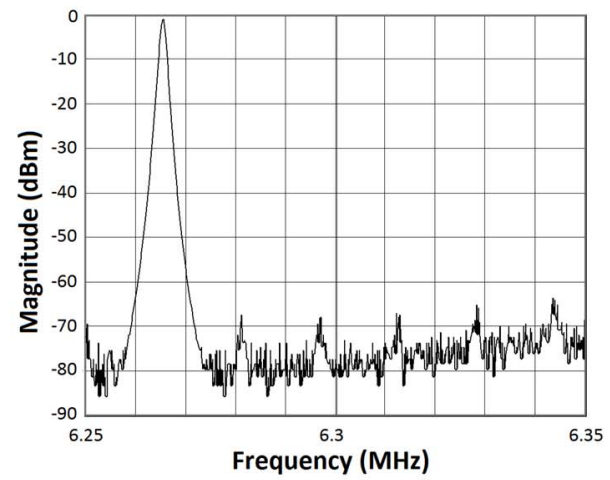

(c)

Fig. 10. Measured output spectra using second order $\Sigma \Delta \mathrm{M}$ and 2-level output. (a) $\Sigma \Delta \mathrm{M}$ with 2-level quantizer. (b) $\Sigma \Delta \mathrm{M}$ with 3-level quantizer. (c) $\Sigma \Delta \mathrm{M}$ with 3-level quantizer and predistortion.

3-level and both 2-level modulators decreased with the OSR as per increase of the quantization noise level. The lower SNR improvement in the case of 9-tone than in 1-tone case was caused by higher level of $E_{\mathrm{EQ}}$ and by higher probability of the input falling into low-amplitude region where the predistortion is less efficient.

\section{EXPERIMENTAL RESULTS}

In order to validate the new approach of linearization, the modulator of Fig. 1(a) was implemented using a second order CIFB $\Sigma \Delta$ M. The input signal was a single tone of amplitude occupying 0.75 of FS and frequency $f_{0}=0.0025 f_{\Sigma \Delta}$. The modulators were implemented in Spartan 3AN FPGA. The modulator was supplied with low phase jitter (0.5 ps rms from $12 \mathrm{kHz}$ to $12.5 \mathrm{MHz}$ ) oscillator of frequency $f_{\mathrm{CLK}}=25 \mathrm{MHz}$. The clock frequency for $\Sigma \Delta \mathrm{M}$ was $f_{\Sigma \Delta}=$ $\left(f_{\mathrm{CLK}} / 4\right)=6.25 \mathrm{MHz}$. All modulators operated using 16bit accuracy. While a pair of 16-bit, second order $\Sigma \Delta \mathrm{M}$ can be implemented using 96 look-up-tables (LUTs), 64 flip-flops and eight adders, the implementation of the linearization in both branches requires 42 LUT, 64 flip-flops and 10 adders. Although the additional hardware needed for linearization is not negligible, the additional power consumption is small. This is because high oversampling ratio used in both $\Sigma \Delta \mathrm{M}$ enables sampling the input signal at a fraction of $f_{\Sigma \Delta}$. A zero-insertion or sample-hold techniques [4], [5] can be used to reduce power consumption of the linearization by factor of 4,8 , or more.

Three different configurations of the modulator were implemented for performance comparison. The output spectrum of the modulator employing a basic 2-level LP $\Sigma \Delta \mathrm{M}$ is shown in Fig. 10(a). The output from modulator employing 3-level $\mathrm{LP} \Sigma \Delta \mathrm{M}$ and followed by 2-level quantizer is shown in Fig. 10(b). As expected, the change from 2-level to 3-level $\Sigma \Delta \mathrm{M}$ results in the reduction of the noise floor is by approximately $6 \mathrm{~dB}$. In addition, the undesired artifacts at frequencies $f_{\Sigma \Delta}+f_{0}$ and $f_{\Sigma \Delta}+2 \cdot f_{0}$ caused by 2-level quantizer are also observed in the output spectrum. The output spectrum of Fig. 10(c) is free of the nonlinear effects and it shows an SNR improvement by $6 \mathrm{~dB}$ when comparing with Fig. 10(a). The improvement has been achieved with linearization blocks operating at sampling frequency $(1 / 4) f_{\Sigma \Delta}$

\section{CONCLUSION}

This brief analyzed a band pass modulation technique utilizing 3-level $\Sigma \Delta \mathrm{M}$ followed by 2-level quantizer, aiming in elimination of nonlinear effects caused by conversion from 3-level to 2-level output. It has been demonstrated that with the technique derived in this brief, a $22 \mathrm{~dB}$ cancellation of the error resulting from nonlinear effects is possible. This technique is suitable for use in the modified first and second order $\Sigma \Delta \mathrm{M}$, whose 2-level output can show up to $6 \mathrm{~dB}$ higher SNR than equivalent, conventional 2-level BP $\Sigma \Delta$ M. The SNR improvement in $\Sigma \Delta \mathrm{M}$ is achieved at a cost of a small power consumption increase, while no increase of LP $\Sigma \Delta \mathrm{M}$ sampling frequency is required. This technique can be suitable in alldigital transceivers [6], where reduction of quantization noise power is of importance. It can be also used to further enhance SNR in the time-interleaved modulators [4].

\section{REFERENCES}

[1] T. Podsiadlik, J. Dooley, and R. Farrell, "Improved coding-efficiency two-level source encoder for RF switch-mode power amplifiers," IEEE Trans. Circuits Syst. II, Exp. Briefs, vol. 59, no. 10, pp. 618-622, Oct. 2012.

[2] S. R. Norsworthy, R. Schreier, and G. C. Temes, Delta-Sigma Data Converters: Theory, Design, and Simulation. Piscataway, NJ, USA: IEEE Press, 1997.

[3] T. Podsiadlik, J. Dooley, and R. Farrell, "Parallelization of bandpass sigma-delta modulators for class-S digital power amplifiers," in Proc. Conf. Ph.D. Res. Microelectron. Electron. (PRIME), Berlin, Germany, 2010, pp. 1-4.

[4] T. Podsiadlik and R. Farrell, "Time-interleaved $\Sigma \Delta$ modulators for FPGAs," IEEE Trans. Circuits Syst. II, Exp. Briefs, vol. 61, no. 10, pp. 808-812, Oct. 2014.

[5] M. Kozak and I. Kale, Oversampled Delta-Sigma Modulators: Analysis, Applications, and Novel Topologies. Boston, MA, USA: Kluwer Acad., 2003.

[6] R. F. Cordeiro, A. S. R. Oliveira, and J. M. N. Vieira, "All-digital transmitter with a mixed-domain combination filter," IEEE Trans. Circuits Syst. II, Exp. Briefs, vol. 63, no. 1, pp. 4-8, Jan. 2016. 